\title{
Log-Domain Time-Multiplexed Realization of Dynamical Conductance-Based Synapses
}

\author{
Theodore $\mathrm{Yu}^{1,3}$ and Gert Cauwenberghs ${ }^{2,3}$ \\ ${ }^{1}$ Department of Electrical and Computer Engineering, Jacobs School of Engineering \\ ${ }^{2}$ Department of Bioengineering, Jacobs School of Engineering \\ ${ }^{3}$ Institute for Neural Computation \\ University of California San Diego, La Jolla, CA 92093
}

\begin{abstract}
We present a compact circuit architecture for analog VLSI realization of event-addressable neuromorphic arrays with conductance-based synaptic dynamics. Synaptic input events are time-multiplexed, pooled by synapse type according to common reversal potential and activation dynamics. One such physical synapse element per postsynaptic neuron is provided for each type, selected by type index along with postsynaptic address. A log-domain encoding of first-order linear dynamics of synaptic conductance results in a compact circuit realization with three MOS transistors per synapse element. Circuit simulations show low-power operation with linear dynamics in conductance.
\end{abstract}

\section{INTRODUCTION}

Neuromorphic engineering [1] utilizes inspiration from biology and neurobiology to direct and motivate the design and modeling of circuits and systems. By emulating form and architecture in biological systems, neuromorphic engineering seeks to emulate function as well. Investigation of neural behavior on large scale requires efficient and realistic modeling and implementation of neurons and their synaptic connections [2]-[4].

Analysis of a variety of different implementations of conductance-based dynamical synapses, and new circuit that overcomes some of their limitations, are presented in [5]. In particular, [5] analyzes the trade-offs among the different implementations regarding functionality of the temporal dynamics and the required layout size, and offers a circuit with linear dynamics in conductance. Earlier implementations of VLSI synapses such as the pulse current-source synapse [1] and reset-and-discharge synapse [6] suffer from inability to integrate input spikes into continuous output currents and linearly sum postsynaptic currents respectively. Other previous circuits such as the linear charge-and-discharge synapse [7] and current-mirror-integrator synapse [8], [9] , and [10] also suffer from nonlinear summation of postsynaptic currents. The synapse implementations of log-domain integrator synapse [11] and diff-pair integrator synapse [5] implement linear summation of postsynaptic currents, but they require an $M_{w}$ p-FET or additional transistors.

Here we show that a variant on the log-domain implementation gives rise to linear conductance dynamics in more compact form. Here we present a three-transistor realization of a dynamical conductance-based synapse element, serving

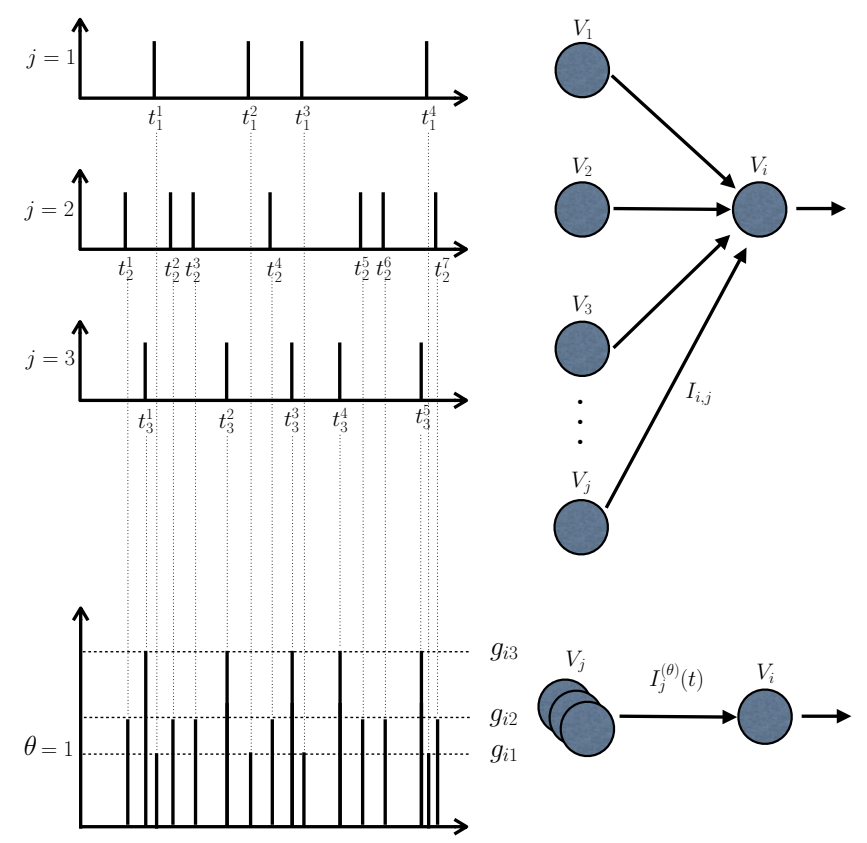

Fig. 1. Pooling of synapses with common reversal potential and activation dynamics, but possibly with different conductances, by time-multiplexing input events from $j$ presynaptic neurons.

multiple synapses with common reversal potential and activation dynamics. The time-multiplexing synapse element pools spike input events from multiple presynaptic source addresses through the address-event representation (AER, [12]) communication framework as seen in Fig. 1.

\section{SynAPSe ARray ARChitecture}

This paper focuses upon the architectural design of the pooled synapse input for each neuron within the neural array. We assume that the number of distinct synapse types is limited to a relatively low number $k, e . g$., $k=8$. This assumption is typically valid even in large-scale cortical models. We pool synapses of the same type serving the same postsynaptic terminal into a time-multiplexed synapse element. Synapse elements in the array are activated by presynaptic events presented through an AER input interface [12]. Neurons receiving 
(a)

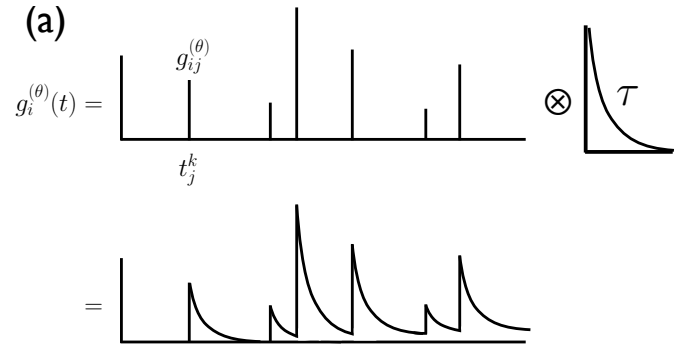

(b)

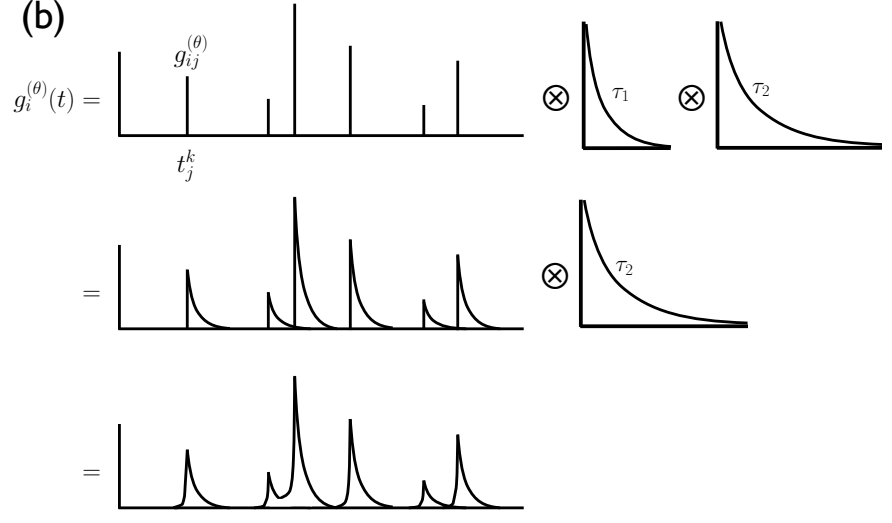

Fig. 2. Illustration of the convolution between the conductance dynamics and conductance strength using two versions (a) a single decay $\tau$, and (b) a rise and fall time $\tau_{1}$ and $\tau_{2}$.

synaptic inputs from these elements further interface through AER arbitration to generate postsynaptic output events [12].

\section{SyNAPSE ELEMENT}

\section{A. Modeling of Conductance Dynamics}

We assume a general conductance-based synapse with continuous activation dynamics. The postsynaptic membrane receives synaptic current contributions,

$$
\sum_{j} I_{i j}=\sum_{j} \sum_{k} g_{i j} f_{i j}\left(t-t_{j}^{k}\right)\left(V_{i}-E_{i j}\right)
$$

where $i$ denotes the post-synaptic neuron, $j$ denotes the presynaptic neuron, $k$ indicates the spiking event number, $g_{i j}$ is the conductance strength between neuron $i$ and neuron $j$, $f_{i j}\left(t-t_{j}^{k}\right)$ indicates the conductance dynamics profile, $V_{i}$ is the membrane voltage of pre-synaptic neuron $i$, and $E_{i j}$ is the reversal potential between neuron $i$ and neuron $j$.

Synaptic current contributions to postsynaptic neuron $i$ are partitioned according to synapse type as

$$
\sum_{j} I_{i j}=\sum_{j} I_{i j}{ }^{(1)}+\sum_{j} I_{i j}{ }^{(2)}+\ldots+\sum_{j} I_{i j}{ }^{(k)}
$$

where each partition serves synapses with common synapse parameters in terms of reversal potentials

$$
E_{i j}^{(\theta)}=E^{(\theta)} \forall i, j
$$

and activation dynamics

$$
f_{i j}^{(\theta)}=f^{(\theta)} \forall i, j
$$

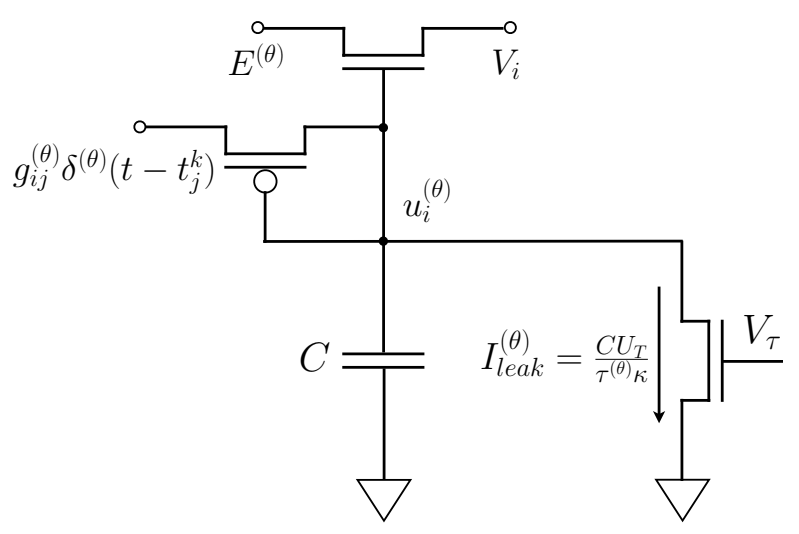

Fig. 3. The circuit implementing the synapse element consisting of 3 MOS transistors.

where $\theta$ indicates the synapse type. The partitions pool each of the synaptic contributions from the respective presynaptic neurons as:

$$
\begin{aligned}
\sum_{j} I_{i j}^{(\theta)} & =\sum_{\theta} \sum_{j} \sum_{k} g_{i j}^{(\theta)} f^{(\theta)}\left(t-t_{j}^{k}\right)\left(V_{i}-E^{(\theta)}\right) \\
& =\sum_{\theta} \sum_{k} g_{i}^{(\theta)}(t)\left(V_{i}-E^{(\theta)}\right)
\end{aligned}
$$

where $g_{i}^{(\theta)}(t)$ denotes the time-multiplexed pooled conductance of synapse element $(\theta)$ of postsynaptic neuron $i$ :

$$
g_{i}^{(\theta)}(t)=\sum_{j} g_{i j}^{(\theta)} f^{(\theta)}\left(t-t_{j}^{k}\right) .
$$

The temporal profile of $g_{i}^{(\theta)}(t)$ is illustrated in Fig. 2. A logdomain recurrence relation expressing this pooled conductance leads to compact realization as described next.

\section{B. Linear and Log-Domain Recurrence Relation}

A general conductance dynamics profile $f^{(\theta)}$ can be characterized by two terms: a fall time $\tau_{1}^{(\theta)}$ and a rise time $\tau_{2}^{(\theta)}$. We start by modeling the transient conductance dynamics as a single decaying exponential with time constant $\tau^{(\theta)}$ as illustrated in Fig. 2(a), and note that the more general case can be implemented by convolution of the activation functions $g_{i j}^{(\theta)}$ with decaying exponential on shorter time scale as illustrated in Fig. 2(b). The convolution between the conductance dynamics and conductance strength using a single delay $\tau^{(\theta)}$ is expressed as:

$$
\left(\tau^{(\theta)} \frac{d}{d t}+1\right) g_{i}^{(\theta)}(t)=\sum_{j} g_{i j}^{(\theta)} \delta\left(t-t_{j}^{k}\right)
$$

where $\delta\left(t-t_{j}^{k}\right)$ is an impulse centered at time $t_{j}^{k}$, representing a presynaptic input event from neuron $j$ of synapse type $\theta$ to postsynaptic neuron $j$.

We utilize a log-domain circuit to exploit the linear relationship between the subthreshold MOSFET gate-source voltage 
and channel currents. So we express $g_{i}^{(\theta)}$ in the log-domain:

$$
\begin{aligned}
u_{i}^{(\theta)} & =\log g_{i}^{(\theta)}(t) \\
\frac{d}{d t} u_{i}^{(\theta)} & =\frac{1}{g_{i}^{(\theta)}(t)} \frac{d}{d t} g_{i}^{(\theta)}(t)
\end{aligned}
$$

leading to

$$
\tau^{(\theta)} \frac{d}{d t} u_{i}^{(\theta)}+1=\sum_{j} \frac{g_{i j}^{(\theta)}(t)}{g_{i}^{(\theta)}(t)} \delta\left(t-t_{j}^{k}\right) .
$$

The solution to the integrator with constant delay (10) in between events $t_{j}^{k}$ and $t_{j}^{k+1}$ is:

$$
u_{i}^{(\theta)}(t)=u_{i}^{(\theta)}\left(t_{j}^{k}\right)-\frac{t-t_{j}^{k}}{\tau^{(\theta)}}, t_{j}^{k}<t<t_{j}^{k+1}
$$

and at the arrival of an event $t_{j}^{k}$, for $\epsilon \rightarrow 0$ :

$$
u_{i}^{(\theta)}\left(t_{j}^{k}+\epsilon\right)=u_{i}^{(\theta)}\left(t_{j}^{k}-\epsilon\right)+g_{i j}^{(\theta)} e^{-u_{i}^{(\theta)}\left(t_{j}^{k}-\epsilon\right)} .
$$

Transformed back to the current domain, the resulting conductance $g_{i}^{(\theta)}$ follows the desired linear dynamics in input activation:

$$
g_{i}^{(\theta)}\left(t_{j}^{k}+\epsilon\right)=g_{i}^{(\theta)}\left(t_{j}^{k}-\epsilon\right)+g_{i j}^{(\theta)}
$$

and exponential decaying conductance in between presynaptic events with time constant:

$$
g_{i}^{(\theta)}(t)=g_{i}^{(\theta)}\left(t_{j}^{k}\right) e^{-\left(t-t_{j}^{k}\right) / \tau^{(\theta)}}, t_{j}^{k}<t<t_{j}^{k+1} .
$$

\section{Circuit Architecture}

The common reversal potential parameter for each synapse partition $E^{(\theta)}$ is simply implemented as a single nMOS transistor operating in the subthreshold region:

$$
I_{N M O S}=\lambda I_{0} e^{\kappa_{n} V_{g} / U_{T}}\left(e^{-V_{s} / U_{T}}-e^{-V_{d} / U_{T}}\right),
$$

where $V_{g}$ is the gate voltage, $V_{s}$ is the source voltage, $V_{d}$ is the drain voltage, $\lambda$ is the $\mathrm{W} / \mathrm{L}$ ratio of the transistor, $I_{0}$ is the subthreshold pre-exponential current factor, $\kappa_{n}$ indicates the back gate effect, and $U_{T}$ is the thermal voltage, $k T / q$. The transistor operates in the subthreshold region while the drain-to-source voltage is less than $4 U_{T}$. Since the voltages are implemented in log-domain circuits, the resulting output current can be expressed as:

$$
I \propto \kappa V_{u}\left(V_{i}-E^{(\theta)}\right),
$$

To implement the input recurrence (12) composed of the input term of the incoming conductance strength value $g_{i j}^{(\theta)}$ multiplied by a negative exponential $e^{-u_{i}^{(\theta)}\left(t_{j}^{k}\right)}$, we utilize CMOS technology to implement the negative exponential $e^{-u_{i}^{(\theta)}\left(t_{j}^{k}\right)}$ with a single diode-connected pMOS transistor operating in the subthreshold region:

$$
I_{P M O S} \propto e^{-\kappa_{p} V_{g} / U_{T}},
$$

We activate the pMOS with a short pulse centered at $t_{j}^{k}$. The conductance strength $g_{i j}^{(\theta)}$ can in principle be implemented by modulating the pulse voltage logarithmically. Rather than
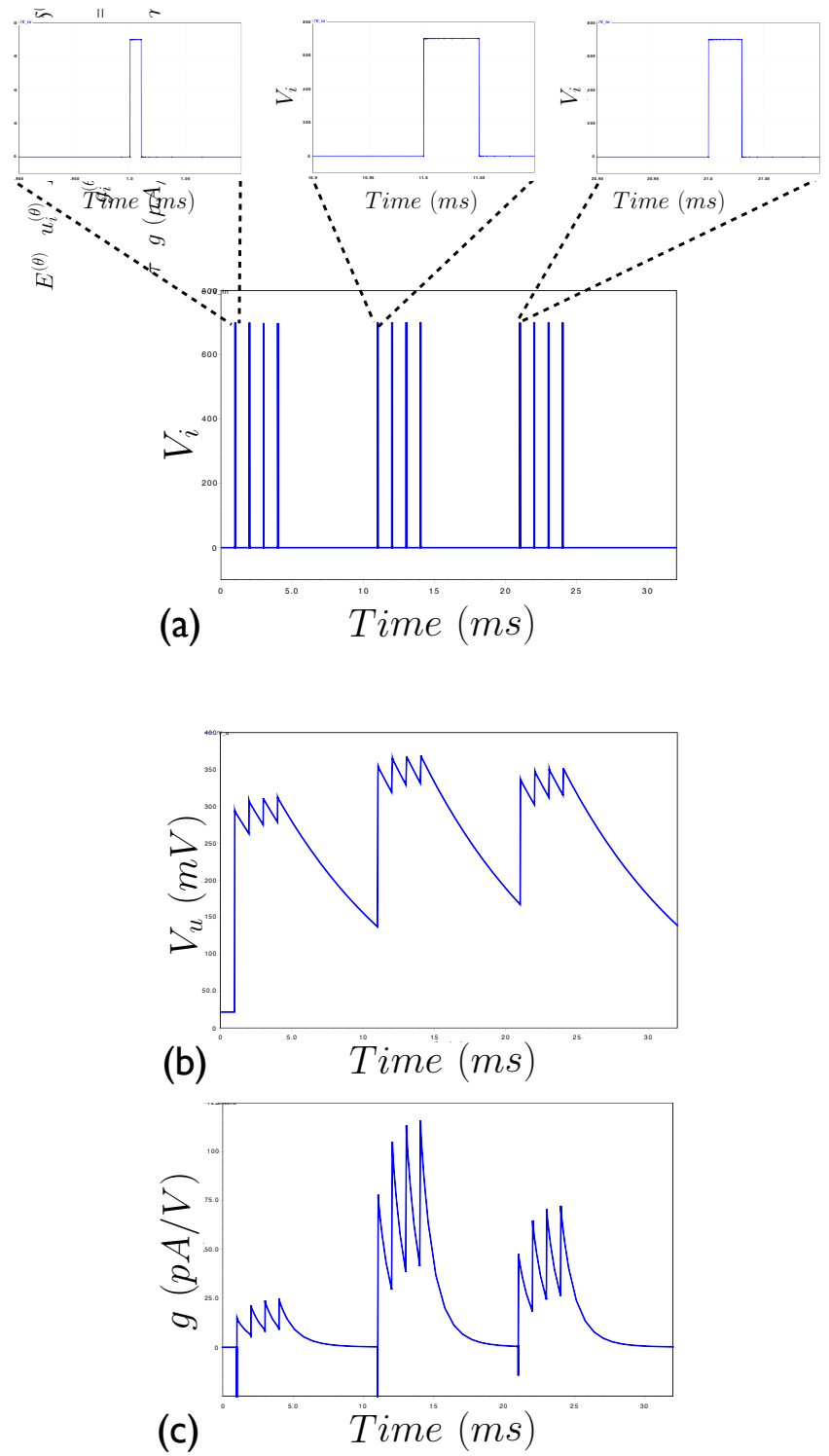

Fig. 4. Transistor-level circuit simulation illustrating both the: a) activation function $g_{i}^{(\theta)}$ with 3 groups of different activation widths (detail shown in inset); b) log-domain variable $u$ and c) time-domain conductance $g$.

adding this complication to the circuit and the drivers at the periphery of the array, we modulate the pulse width linearly in the conductance strength $g_{i j}^{(\theta)}$. Notice that a back gate effect parameter $\kappa_{n}$ and $\kappa_{p}$ is present in both of the expressions for the input (13) and output (15) of the synapse element. The $\kappa$ parameter indicates the efficiency of a change the gate voltage and the resultant change in surface potential. This loss in efficiency is due to the bulk terminal in a MOSFET, which can act as another gate terminal (also referred to as the 'back-gate effect'). Fortunately, this effect will have little consequence if the nMOS and pMOS devices have sufficiently close back-gate effects, $\kappa_{n} \approx \kappa_{p}$.

By virtue of the log-domain transformation, the decaying exponentials $e^{-t / \tau}$ in the conductance dynamics $f_{i j}\left(t-t_{j}^{k}\right)$ are implemented using a single nMOS transistor operated in 


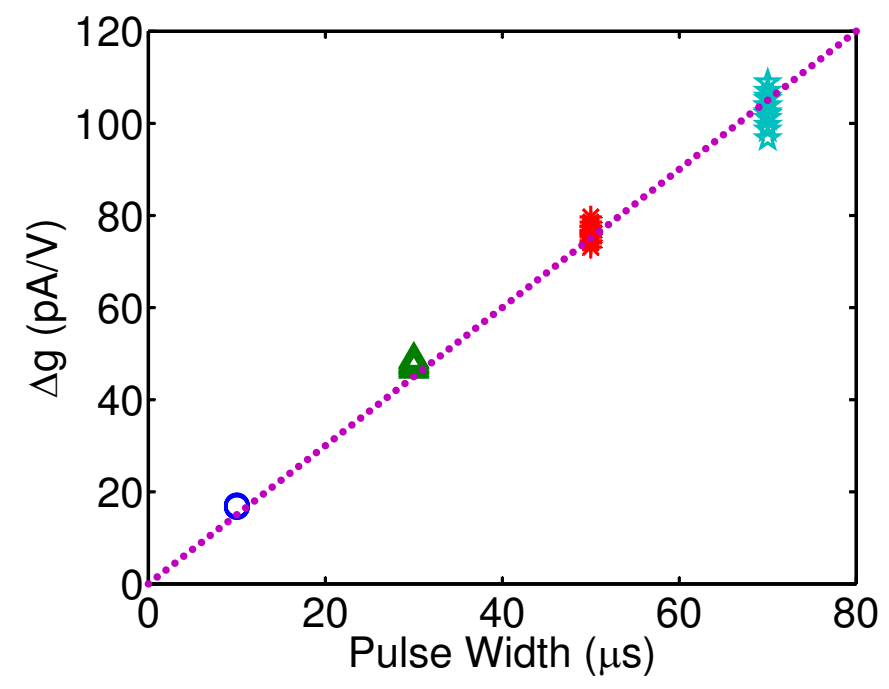

Fig. 5. Transistor-level circuit simulation illustrating $\Delta g$ for 4 groups of different activation widths.

subthreshold and used as a constant current source to linearly charge capacitor $C$. As shown in Fig. 2(b), the conductance dynamics $f_{i j}\left(t-t_{j}^{k}\right)$ can be extended to a rise time $\tau_{1}$ and fall time $\tau_{2}$ through convolution. This could be accomplished by driving the source of the pMOS with a sequence of pulses. The complete dynamical conductance-based synapse circuit implementation is shown in Fig. 3. The circuit is compact, requiring only 3 transistors to implement.

\section{CHARACTERIZATION}

To verify the conductance dynamics, we performed transistor-level simulations (using Spectre and parameters of a $0.13 \mu \mathrm{m}$ CMOS process) of the synapse circuit driven by a train of presynaptic impulses, modulated with three different pulse widths, with relative magnitudes 1,3 , and 5 , emulating the effect of three time-multiplexed pooled synapses. The circuit output in response to the sequence of input synaptic events is shown in Fig. 4.

To verify the linearity of postsynaptic conductance in presynaptic activation, we studied the dependence of the conductance time profile as a function of pulse width and pulse interval. We observed the step in conductance $\Delta g$ for a train of pulses at variable pulse intervals, for four different values of pulse width with relative magnitudes $1,3,5$, and 7 as shown in Fig. 5. The four distinct and compact groups for each of the four different activation widths indicate the linearity of the conductance according to the convolution model (13) and (14). Furthermore, the centers of the clusters for each of the different activation widths are colinear through the origin, confirming linearity in input pulse width.

\section{CONCLUSION}

We have formulated a dynamical conductance-based synapse cell in a compact circuit design. Circuit simulations verify log-domain implementation as well as an output magnitude scaled to the input conductance strength. The circuit implementation is compact, requiring only 3 transistors. This small footprint, coupled with the low-power subthreshold design, make this design a suitable candidate for large-scale implementation of synaptic arrays in addressable neuromorphic systems, with reconfigurable synaptic connectivity as well as individually selectable synaptic dynamics.

\section{ACKNOWLEDGMENT}

This research was supported by NIH/NIA R01AG029681.

\section{REFERENCES}

[1] Mead, C.A., Analog VLSI and Neural Systems, Addison-Wesley, 1989

[2] Serrano-Gotarredona, R.; Oster, M.; Lichtsteiner, P.; Linares-Barranco, A.; Paz-Vicente, R.; Gomez-Rodriguez, F.; Camunas-Mesa, L.; Berner, R.; Rivas-Perez, M.; Delbruck, T.; Shih-Chii Liu; Douglas, R.; Hafliger, P.; Jimenez-Moreno, G.; Ballcels, A.C.; Serrano-Gotarredona, T.; AcostaJimenez, A.J.; Linares-Barranco, B. "CAVIAR: A 45k Neuron, 5M Synapse, 12G Connects/s AER Hardware SensoryProcessing LearningActuating System for High-Speed Visual Object Recognition and Tracking", IEEE Trans. Neural Networks, vol. 20(9), pp. 1417-1438, 2009.

[3] Serrano-Gotarredona, R.; Serrano-Gotarredona, T.; Acosta-Jimenez, A.; Serrano-Gotarredona, C.; Perez-Carrasco, J.A.; Linares-Barranco, B.; Linares-Barranco, A.; Jimenez-Moreno, G.; Civit-Ballcels, A. "On RealTime AER 2-D Convolutions Hardware for Neuromorphic Spike-Based Cortical Processing", IEEE Trans. Neural Networks, vol. 19(7), pp. 11961219, 2008.

[4] Badoni, D.; Giulioni, M.; Dante, V.; Del Giudice, P. "An aVLSI recurrent network of spiking neurons with reconfigurable and plastic synapses", Proc. IEEE ISCAS 2006, pg. 1227-1230, 2006.

[5] Bartolozzi, C.; Indiveri, G. "Synaptic Dynamics in Analog VLSI", Neural Computation, vol. 19(10), pp. 2581-2603, 2007.

[6] Lazzaro, J. P., "Low-power silicon axons, neurons, and synapses", in Zaghloul, M.E.; Meador, J.L.; and Newcomb, R.W. (Eds.), Silicon implementation of pulse coded neural networks, Norwell, MA: Kluwer, pp. 153164, 1994.

[7] Arthur, J.; Boahen, K. "Recurrently connected silicon neurons with active dendrites for one-shot learning", IEEE International Joint Conference on Neural Networks, vol. 3, pp. 1699-1704, 2004.

[8] Boahen, K.A. "Retinomorphic vision systems: Reverse engineering the vertebrate retina", Unpublished doctoral dissertation, California Institute of Technology, 1997.

[9] Hynna, K.; Boahen, K. "Space-rate coding in an adaptive silicon neuron", Neural Networks, vol. 14, pp. 645656, 2001.

[10] Chicca, E. "A neuromorphic VLSI system for modeling spike-based cooperative competitive neural networks", Unpublished doctoral dissertation, ETH Zurich, Zurich, Switzerland, 2006.

[11] Merolla, P.; Boahen, K. "A recurrent model of orientation maps with simple and complex cells" in Thrün, S.; Saul, L.K., Schölkopf, B. (Eds.), Advances in neural information processing systems, MIT Press. Cambridge, MA, vol. 16, pp. 995-1002, 2004.

[12] Boahen, K. "Point-to-point connectivity between neuromorphic chips using address events", IEEE Trans. on Circuits and Systems II, vol. 47, pp. 416-434, 2000.

[13] Goldberg, D.H.; Cauwenberghs, G.; and Andreou, A.G. "Probabilistic Synaptic Weighting in a Reconfigurable Network of VLSI Integrate-andFire Neurons", Proc. IEEE Int. Symp. Circuits and Systems, vol. 3, pp. 241-244, 2001

[14] Vogelstein, R.J.; Mallik, U.; Vogelstein, J.T.; and Cauwenberghs, G. "Dynamically Reconfigurable Silicon Array of Spiking Neurons With Conductance-Based Synapses”, IEEE Trans. Neural Networks, vol. 18(1), pp. 253-265, 2007.

[15] Vogelstein, R.J.; Mallik, U.; Culurciello, E.; Cauwenberghs, G.; and Etienne-Cummings, R. "Saliency-Driven Image Acuity Modulation on a Reconfigurable Silicon Array of Spiking Neurons", Adv. Neural Information Processing Systems (NIPS'2004), Cambridge: MIT Press, vol. 17, 2005.

[16] Destexhe, A.; Mainen, Z.F.; and Sejnowski, T.J. "Synthesis of Models for Excitable Membranes, Synaptic Transmission and Neuromodulation Using a Common Kinetic Formalism,” J. Comp. Neuroscience, vol. 1, pp. 195-230, 1994. 